\title{
Novel Steroid-Sensing Model and Characterization of Protein Interactions Based on Fluorescence Anisotropy Decay
}

\author{
Ko-Shing Chang, ${ }^{\ddagger}$ Liyang Luo, ${ }^{\ddagger}$, Chih-Wei Chang, ${ }^{\ddagger}$ Yen-Chieh Huang, ${ }^{\ddagger}$ Chih-Yu Cheng, \\ Chen-Shiung Hung, ${ }^{\S}$ Eric Wei-Guang Diau, ${ }^{*,+}$ and Yaw-Kuen $\mathrm{Li}^{*}$, \\ Department of Applied Chemistry and Institute of Molecular Science, National Chiao Tung University, \\ Hsinchu 30010, Taiwan, and Institute of Chemistry, Academia Sinica, Taipei 11529, Taiwan
}

Received: September 03, 2009; Revised Manuscript Received: February 03, 2010

\begin{abstract}
Intramolecular binding of a ligand with an alkyl link, $\left(-\mathrm{CH}_{2}\right)_{3}$, covalently bound to a residue near the active site of the protein forms a novel steroid-sensing model. A genetically engineered $\triangle^{5}$-3-ketosteroid isomerase (KSI) was designed to conjugate uniquely with this ligand at its Cys-86 through the formation of a disulfide bond. The steady-state protein-ligand binding, mediated by hydrophobic interactions, was confirmed with fluorescence spectra, and the fluorophore-labeled peptide sequence was identified with tandem mass spectra. A comparison of steady-state fluorescence spectra of various fluorophore-labeled KSI mutants reveals that the emission characteristics vary with environmental factors. An evaluation of the decay of the fluorescence anisotropy of the fluorophore indicates the existence of an intramolecular protein-ligand binding interaction. The measurement of time-resolved fluorescence anisotropy of various protein-ligand complexes yielded values of anisotropy decay representing the degrees of freedom of the fluorophore related to its location, inside or outside the steroid-binding domain. When 19-norandrostenedione (19-NA) was added to this protein-ligand system, competitive binding between the ligand and the steroid was observed; this finding confirms the feasibility of the design of steroid detection with engineered KSI. On integration of this protein-ligand system with a silicon-based nanodevice (a p-type field-effect transistor with an ultrathin body), a noncharged steroid, 19-NA, became detectable at a micromolar level (Biosens. Bioelectron. 2008, 23, 1883).
\end{abstract}

\section{Introduction}

The interaction of a protein with a ligand becomes understood by evaluating how the protein structure influences the thermodynamic parameters of the binding ligand. Not only hydrophobic interactions but also differences in hydrogen bonding, van der Waals, and electrostatic interactions, as well as conformational modifications, determine the thermodynamics of these interactions. The binding affinity of protein-ligand ${ }^{1,2}$ and proteintransition-state complexes is reviewed elsewhere. ${ }^{3}$ Insight into these aspects is valuable for the design of probes for chemical and biochemical recognition. ${ }^{4-6}$

An intramolecular protein-ligand system with the binding moieties of a ligand connected to a protein ${ }^{7}$ was constructed with a benzenesulfonamide derivative covalently attached to the surface of a human carbonic anhydrase II through an oligo(ethanediol) link. Although the system was designed for investigating a correlation of the length of the link with the effective molarity of the ligand, it also indicated a prospective application of biorecognition using a model of intramolecular protein-ligand interaction.

We discuss herein the application of a steroid-recognition system constructed to incorporate an intramolecular protein-ligand interaction. An investigation of this system might lead to a design of a biosensor for sensitive detection of steroids, which is important because of their diverse biological functions. In

* To whom correspondence should be addressed. E-mail: Y.-K.L., yk1@cc.nctu.edu.tw; W.-G.D.,diau@mail.nctu.edu.tw.

\$ Abbreviations: 5-[(2-aminoethyl)amino]-1-naphthalenesulfonic acid, 1,5-EDANS; 5-[(2-(iodoacetamido)ethyl)amino]naphthalene-1-sulfonic acid, I-14; $\triangle^{5}$-3-ketosteroid isomerase, KSI; 19-norandrostenedione, 19-NA.

* National Chiao Tung University.

$\S$ Academia Sinica. pharmaceutical, physiological and clinical tests, steroid assays are based on complicated chemical or enzyme catalytic reactions monitored with chromatographic, ${ }^{8-10}$ electrochemical, ${ }^{11,12}$ optical, ${ }^{13}$ or mass detection platforms. ${ }^{14,15}$ The production of coating steroid antigens for use in immunoassays is essential for the development of steroid analysis, but the tedious nature and great cost involved in the preparation of a complicated antibody or receptor are among the factors that restrict the development of ELISA-like assays.

A steroid-recognition model, constructed with intramolecular protein-ligand interaction using $\triangle^{5}-3$-ketosteroid isomerase (KSI) and a ligand containing a 5-((2-aminoethyl)amino)-1naphthalenesulfonic acid (1,5-EDANS) moiety, is constructed, as depicted in Scheme 1. The engineered KSI was designed for use as a biological component; the ligand was tethered uniquely to Cys- 86 through a disulfide bond near the steroid-binding site. The major driving force favoring this association is described as a hydrophobic interaction that prompts the 1,5-EDANS moiety to bind with the protein. The thermodynamics of this protein-ligand binding can be altered with a favorable control of enthalpy and, particularly in this model, the characteristic "entropy-driven" thermodynamic signature of the steroid. The presence of a steroid at varied concentrations can affect the binding ratio of this ligand with KSI. A decreased binding ratio of the ligand results in an increased quantity of the ligand being pushed from the steroid-binding site.

The development of an ideal fluorescence probe system to explore molecular recognition in biological environments is a useful approach to investigate protein interactions. ${ }^{4-6}$ The $1,5-$ EDANS moiety, functioning as both ligand and fluorescence probe, was employed for quantitative analysis of the protein-ligand 
SCHEME 1: Chemical Structures of Ligands and the Steroid-Recognition Model

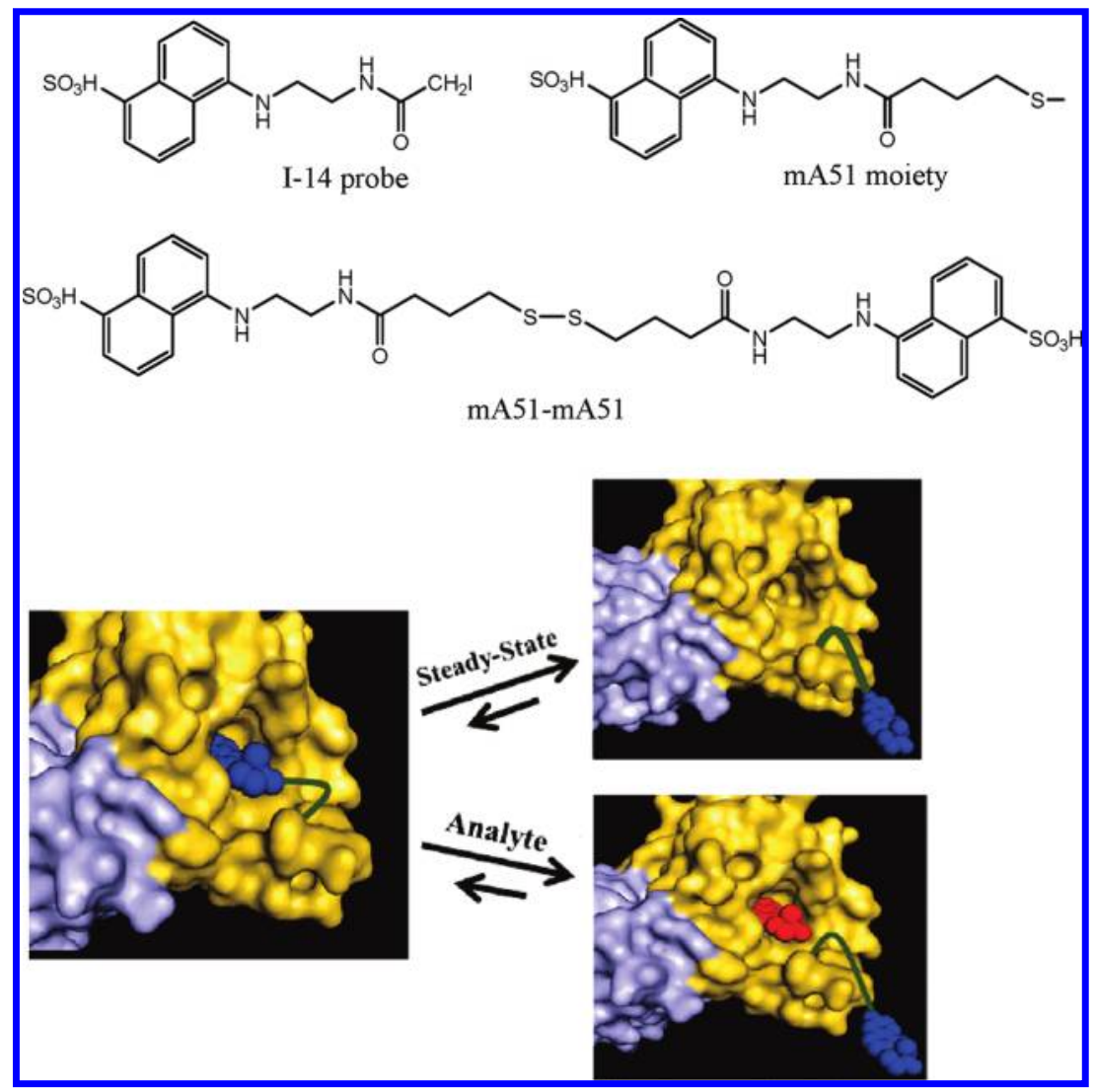

interactions based on measurements of the fluorescence quantum yield, depopulation dynamics and depolarization kinetics. We measured time-resolved fluorescence anisotropy to examine the construction of a steroid-recognition model by determining the environmental effect of the fluorescence probe. The anisotropy decays monitor the rotation of the emission dipole during the lifetime of the excited state; the method thus provides information about the local motion of the fluorophore, segmental mobility, and the rotational period of an entire macromolecule. We applied this steroid-recognition model on a silicon-based nanodevice (a p-type field-effect transistor with an ultrathin body) to detect 19 -norandrostenedione (19-NA). ${ }^{16}$

\section{Experiments}

2.1. Design of KSI Mutants. Wild-type KSI is a dimeric protein containing in each subunit 125 amino-acid residues but no cysteine and tryptophan. Much research has been performed on KSI regarding the catalytic mechanism, the identification of essential groups, ${ }^{17-19}$ steady-state and time-resolved spectra, ${ }^{20,21}$ and protein structure. ${ }^{22}$

To eliminate the possibility of multilabeling and a complicated orientation evolving from ligand conjugation and further applications, we incorporated into the wild-type protein eight mutant sites: Y55F, K60R, F86C, F88G, K92R, K108R, K119R, and $\mathrm{A} 125 \mathrm{~K}$. In the following text this engineered protein is designated as Art_KSI. The precursor of the ligand, named mA51-mA51 (Scheme 1), was synthesized on coupling two molecules of 1,5-EDANS with 4,4'-dithiodibutanoic acid. The Cys-86 residue, which is near the steroid-binding site, was designed to react with mA51-mA51 through a thiol substitution to form a disulfide bond between protein and the ligand (mA51); the resulting protein is called Art_KSI/mA51. Two other KSI mutants were constructed: $\mathrm{Y} 55 \mathrm{~F} / \mathrm{Y} 88 \mathrm{~F}$ is a double-site mutant (named Y14only_KSI), and Y55F/Y88F/D38N/D99N/126C is a mutant with multiple sites and an extra Cys added at the C-terminus of the polypeptide (named mKSI_126C). A fluorescence probe, 5-[(2-(iodoacetamido)ethyl)amino]naphthalene1-sulfonic acid (1,5-I-AEDANS, abbreviated as I-14, Scheme 1 ), was used to label individually on an internal residue and an external residue of the steroid-binding site in Y14only_KSI and mKSI_126C, respectively. These two I-14-labeled proteins were employed to simulate the intramolecular interaction of Art_KSI/ mA51 with steady-state and time-resolved fluorescence spectra. The probes were attached extrinsically to positions in KSI, and indicated the dynamics of the probe in the protein through the fluorescence anisotropy decay.

In summary we constructed three KSI mutants: Y14only_KSI, mKSI_126C, and Art_KSI. Two fluorescence probes (I-14 and the synthetic mA51-mA51) served as labeling reagents. The resulting proteins are designated Y14only_KSI/I-14, mKSI_126C/ I-14, and Art_KSI/mA51, correspondingly.

2.2. KSI Gene Construction. The artificial genes of wildtype KSI and Art_KSI were constructed by PCR amplification with forward and reverse primers in four sets. The sequences of primers for Art_KSI gene (not shown for wild-type KSI) appear in Table 1S1, Supporting Information. A fragment of DNA with 375 bp was obtained and inserted into a TA cloning vector (Yeastern Biotech Co. Taiwan). Several positive clones were identified; the plasmid DNA from one clone was digested with Nde I and BamH I. The fragment was subcloned into pRSET A for expression. Y14only_KSI, containing only a single tyrosine residue (Tyr-14) in the protein, was constructed from wild-type KSI with a PCR method (QuikChange, Stratagene Co.). mKSI_126C was constructed through multistep mutagenesis using the plasmid of Y14only_KSI as DNA template. 
2.3. Protein Expression and Purification. The pRSET vector containing the KSI gene or its mutants were expressed in E. coli strains BL21 (DE3). All proteins were obtained through the following procedures. After induction $\left(16 \mathrm{~h}, 28^{\circ} \mathrm{C}\right)$, a bacterial culture $(1 \mathrm{~L})$ was harvested on centrifugation and further resuspended in phosphate buffer $(15 \mathrm{~mL}, 20 \mathrm{mM}, \mathrm{pH}$ 7.5). Cells were disrupted with ultrasonication. A precipitant containing the crude enzyme was obtained from the supernatant on treating with ammonium sulfate up to $50 \%$ saturation. The crude enzyme was further resuspended in phosphate buffer (10 $\mathrm{mL}, 20 \mathrm{mM}, \mathrm{pH}$ 7.5). After desalting, the sample solution (10 $\mathrm{mL}$ ) was loaded onto a column (HiTrap Q, $30 \mathrm{~mL}$, Pharmacia Co.) for chromatographic separation. The column was eluted with phosphate buffer ( $20 \mathrm{mM}, \mathrm{pH} 7.5$, flow rate $1 \mathrm{~mL} / \mathrm{min}$ ) and a linear gradient of $\mathrm{NaCl}(2.5 \mathrm{mM} / \mathrm{min})$. KSI was eluted in the range $50-75 \mathrm{mM}$ of $\mathrm{NaCl}$. This protocol for protein purification is appropriate for other KSI mutants. The quality of the purified proteins was examined with both SDS-PAGE and LC/MS.

2.4. Kinetic Analysis and Steady-State Fluorescence. The protein concentration was determined as described elsewhere. ${ }^{28}$ Enzyme activity was assayed on measuring the conversion of $\triangle^{5}$-androstene-3,17-dione to $\triangle^{4}$-androstene-3,17-dione in Tris- $\mathrm{HCl}$ $\left(50 \mathrm{mM}, \mathrm{pH} 7.5,25^{\circ} \mathrm{C}\right)$. The reaction was monitored at 248 $\mathrm{nm}$ in the UV spectrum. $K_{\mathrm{m}}$ and $k_{\mathrm{cat}}$ values were estimated from the nonlinear regression of the Michaelis-Menten equation using Grafit program. As Art_KSI contains no tryptophan and only one tyrosine, i.e., Tyr-14, present in the steroid-binding site, Art_KSI exhibits a unique fluorescence emission to describe the protein-steroid binding interaction. The dissociation constant $\left(K_{\mathrm{d}}\right)$ of the KSI-steroid complex was determined from the quenching yield of tyrosine fluorescence. We measured the fluorescence quenching of KSI by steroid on adding KSI to a buffer solution (50 mM Tris- $\mathrm{HCl}, \mathrm{pH} 7.5)$ containing steroid in various amounts. The resulting solution was excited at 278 $\mathrm{nm}$ and fluorescence emission was recorded at $307 \mathrm{~nm}$. Data were analyzed as described in the literature. ${ }^{20}$

2.5. Chemical Modification and Pepsin Digestion of KSI. All labeling reactions were performed at $4{ }^{\circ} \mathrm{C}$ in Tris- $\mathrm{HCl}$ buffer $(50 \mathrm{mM}, \mathrm{pH} 7.5,12 \mathrm{~h})$ containing enzyme $(0.1 \mathrm{mM})$ and labeling reagent $(1 \mathrm{mM})$. The excess labeling reagent was removed with dialysis or ultrafiltration. The efficiency of the labeling reaction was evaluated with LC/MS. For peptide sequencing, KSI $(100 \mu \mathrm{L}, 3.5 \mu \mathrm{g} / \mu \mathrm{L})$ and pepsin $(40 \mu \mathrm{L}, 0.5$ $\mu \mathrm{g} / \mu \mathrm{L}$ in sodium phosphate, $2.0 \mathrm{M}, \mathrm{pH} 1.7)$ were incubated at $25^{\circ} \mathrm{C}$ for $30 \mathrm{~min}$. Control digestion of unlabeled enzyme was performed under identical conditions. The labeled peptide was isolated by HPLC and detected by MS as described below.

2.6. Mass-Spectrometric Analysis. Mass spectra were recorded with a quadrupole time-of-flight mass filter (ESI-Q-TOF, Micromass, Manchester, U.K.). Proteins $(5-10 \mu \mathrm{g})$ used for mass measurement were desalted before analysis. Peptides were separated on a reversed-phase HPLC interfaced with the mass spectrometer. The pepsin hydrolysate of (I-14)-labeled KSI or a control enzyme was loaded onto a $\mathrm{C}_{18}$ column (PLRP-S 100 $\AA$ A $5 \mu \mathrm{m}, 150 \times 4.6 \mathrm{~mm}$, Polymer Laboratories) and eluted with a gradient of acetonitrile $(2-50 \%$, containing $0.02 \%$ formic acid, $60 \mathrm{~min}$, flow rate $0.8 \mathrm{~mL} / \mathrm{min}$ ). A postcolumn flow splitter served to direct $90 \%$ of the sample into a fraction collector; the remainder flowed into the mass spectrometer for analysis. The ESI-Q-TOF system presented a range 100-2000 u of mass-tocharge ratio, $\mathrm{m} / \mathrm{z}$, with scan step $2 \mathrm{~s}$ and interval $0.1 \mathrm{~s} / \mathrm{step}$ between scans. For the quadrupole scan mode the electrospray voltage was $3 \mathrm{kV}$; the block temperature was $80^{\circ} \mathrm{C}$.
2.7. Time-Resolved Fluorescence Anisotropy. Temporally resolved spectra on a picosecond scale were measured with a time-correlated single-photon counting (TCSPC) system (Fluotime 200, PicoQuant); the experimental details are described elsewhere. ${ }^{23}$ A picosecond pulsed-diode laser head (LDH-375, Picoquant) controlled with a picosecond pulsed-laser driver (PDL800-D, Picoquant) provided excitation at $\lambda=375 \mathrm{~nm}$ with fwhm $\sim 70 \mathrm{ps}$. The light from the excitation laser was focused onto the sample holder with a lens; another lens collected the fluorescence emitted from the sample at a right angle. The intensity of the detected signal was attenuated with an iris; a polarizer selected the polarization of the detected fluorescence relative to the excitation laser pulse. A double monochromator compensated the GVD of fluorescence and selected the detection wavelength; the resolution was $8 \mathrm{~nm}$ with slits of width $1 \mathrm{~mm}$. A microchannel-plate photomultiplier was connected to a computer with a TCSPC-module (SPC-630, Becker and Hickl) card for data acquisition. All measurements were made for samples in $50 \mathrm{mM}$ Tris $-\mathrm{HCl}$ at $\mathrm{pH} 7.5$.

\section{Results and Discussion}

3.1. Catalytic Activity of Art_KSI and Binding Affinities toward Steroids. According to LC/MS analysis, the precise molecular mass was $13402 \mathrm{Da}$, consistent with the molecular mass calculated from the amino-acid composition of Art_KSI. The Art_KSI enzyme possesses substantial activity; $k_{\mathrm{cat}} / K_{\mathrm{m}}=$ $1.12 \times 10^{7} \mathrm{M}^{-1} \mathrm{~s}^{-1}$. The dissociation constant $\left(K_{\mathrm{d}}\right)$ of the KSIsteroid complex was determined from the quenching yield of tyrosine fluorescence at $\lambda_{\mathrm{em}}=307 \mathrm{~nm}\left(\lambda_{\mathrm{ex}}=278 \mathrm{~nm}\right)$. For instance, 19-NA interacted with Art_KSI $\left(K_{\mathrm{d}}=10.9 \mu \mathrm{M}\right)$ with binding energy $28.2 \mathrm{~kJ} \mathrm{~mol}^{-1}$. 1,5-EDANS showed much weak binding to Art_KSI with $K_{\mathrm{d}}=350 \mu \mathrm{M}$.

3.2. Identification and Sequencing of the Peptide Labeled with I-14. $\alpha$-Halo acid or amide reagents (such as iodoacetic acid or iodoacetamide) are among those commonly used for thiol modification in protein chemistry. In most proteins, the site of reaction is a cysteine residue, ${ }^{24}$ but wild-type KSI contains no cysteine residue. When Y14only_KSI was treated with I-14, a stoichiometric and covalent label was observed by LC/MS analysis (shown in Figure S1a, Supporting Information). The molecular masses of Y14only_KSI before and after chemical modification are 13672 and $13978 \mathrm{Da}$; the increase of $306 \mathrm{Da}$ confirmed the attachment of the moiety of 5-[(2-(acetamido)ethyl)amino]naphthalene-1-sulfonic acid on KSI. We assume that a specific nucleophile in KSI is accessible for the substitution reaction to proceed. We applied proteolytic digestion combined with extensive LC/MS/MS analysis on sequencing the (I-14)-labeled peptide to resolve the target residue of labeling. The labeled peptides were located within the chromatogram (shown in Figure S1b, Supporting Information) on comparison of the peptides present within digests of labeled and unlabeled Y14only_KSI, using absorption $(335 \mathrm{~nm})$ of I-14 to confirm the locations of the peptides of interest. The doubly charged peptides with $\mathrm{m} / \mathrm{z}=975.8$ and $822.6 \mathrm{u}$ were obtained on RP-HPLC separation of labeled and unlabeled samples, respectively. The mass difference of the two fragments at the singly charged state is $307 \mathrm{Da}$, consistent with the labeling moiety. Both fragments were subjected to MS/MS analysis, yielding spectra shown in Figure 1. The presence of b-ion and $y$-ion unequivocally confirms the sequence of the unlabeled peptide as ATVEDPVTGSEPRSGTAA, corresponding to residues 34-51 of Y14only_KSI. Identification of the point of attachment of the probe moiety was achieved through inspection of the daughter ions of (I-14)-labeled peptide $(\mathrm{m} / \mathrm{z}=975.8 \mathrm{u}$, doubly charged). On comparing a series of b-ion and y-ion 


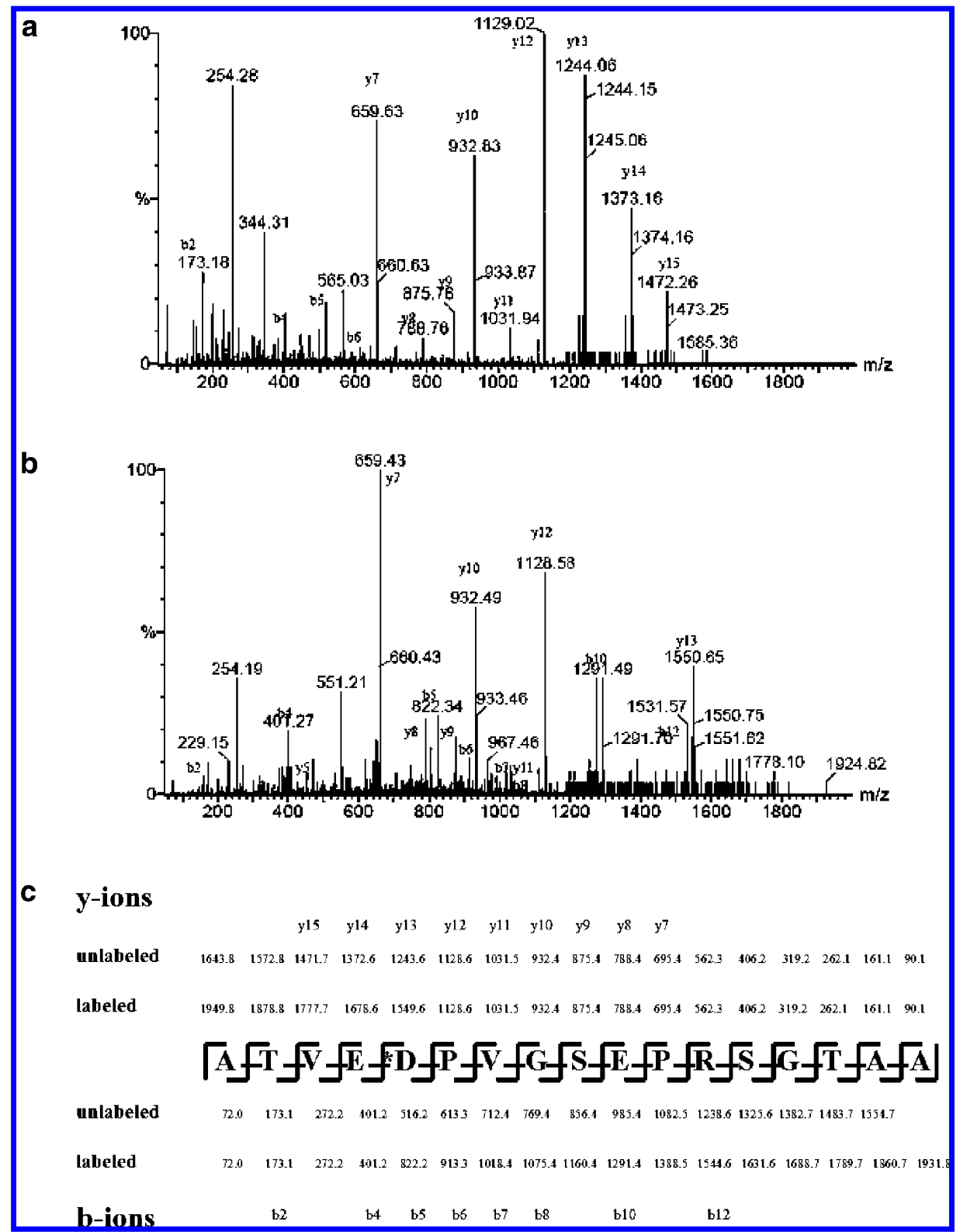

Figure 1. MS/MS analysis of the I-14-labeled peptide. (a) MS/MS daughter-ion spectrum of the unlabeled peptide $(\mathrm{m} / \mathrm{z} 822.6 \mathrm{u}$ in the doubly charged state); (b) MS/MS daughter-ion spectrum of the labeled peptide ( $\mathrm{m} / \mathrm{z}, 975.8 \mathrm{u}$ in the doubly charged state); (c) rationalization of the observed singly charged $y$ - and b-ions with the pattern of the amino-acid sequence of the expected peptide.

derived from both fragments (Figure 1c), we concluded that the moiety of 5-[(2-(acetamido)ethyl)amino]naphthalene-1sulfonic acid was covalently labeled on the Asp-38 residue. Although other effects might assist the labeling reaction of I-14, the hydrophobic nature existing in the active site (i.e., the steroid-binding site) of KSI is considered to play a major role not only promoting the driving force for I-14 binding but also enhancing the nucleophilicity of Asp-38 to react with I-14. A similar nucleophilic reaction is commonly seen in the catalysis of glycoside hydrolase. The side-chain carboxylate of glutamate or aspartate in the active site of glycoside hydrolase functions as a nucleophile to promote the hydrolysis of glycoside through the formation of a glycosyl-enzyme intermediate. ${ }^{25-27}$ The nucleophilicity of the carboxylate group is greater than that in a regular chemical reaction, presumably because of the proximity in the enzyme-substrate complex involving interactions of various types including ionic interaction, hydrogen bond, and hydrophobic interaction.
3.3. Comparison of Fluorescence Polarization Factors of Labeled KSI Mutants. To explore the fluorescence depolarization of I-14 in KSI, we utilized two mutant proteins: Y14only_KSI and mKSI_126C. Depending on the specific labeling residues designed, we readily controlled the fluorophore to label in the steroid-binding site or on the surface of the protein. For instance, mKSI_126C was designed to conduct I-14 labeling on the surface of the protein; this labeling reaction presumably occurs through a nucleophilic attack of Cys-126, which is the C-terminus of the protein and which is structurally predicted to be exposed on the surface. The steady-state emission spectra of I-14 in various solvents were measured to mimic various environmental conditions; the results appear in Figure 2. Samples of I-14 in Tris buffer and in isopropyl alcohol (IPA) served to simulate the hydrophilic (0\% IPA) and hydrophobic (100\% IPA) environments, respectively. In all cases, the absorption was centered at $\sim 335 \mathrm{~nm}$, but emission spectra of I-14 in Tris buffer and in IPA differed significantly: in the 


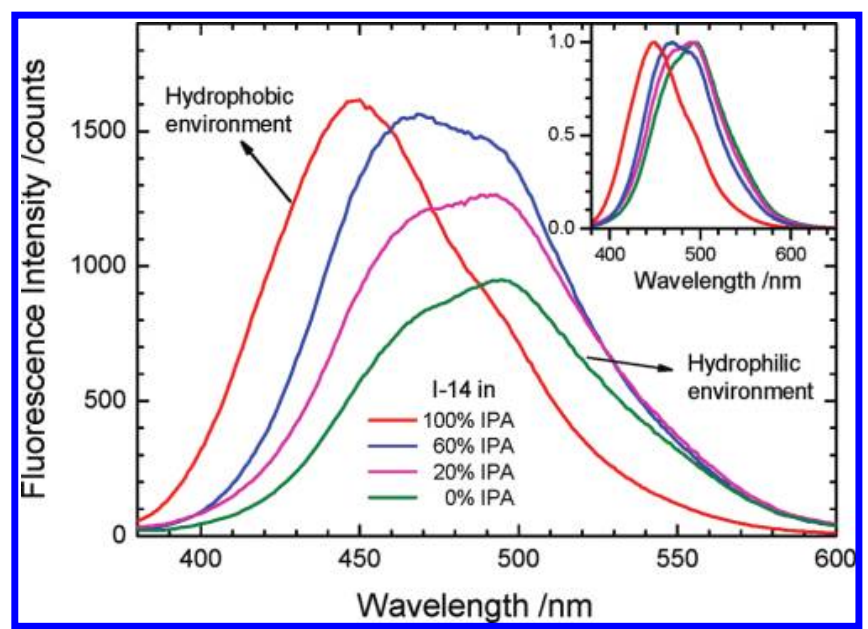

Figure 2. Fluorescence spectra of $\mathrm{I}-14$ in various hydrophobic environments. I-14 was excited at $\sim 335 \mathrm{~nm}$ in $100 \%$ IPA solution (red curve), 60\% IPA solution (blue curve), 20\% IPA solution (magenta curve), and only Tris solution (olive curve). $\mathrm{H}_{2} \mathrm{O}$ compensated the fraction of IPA. The inset shows the corresponding normalized fluorescence spectra.

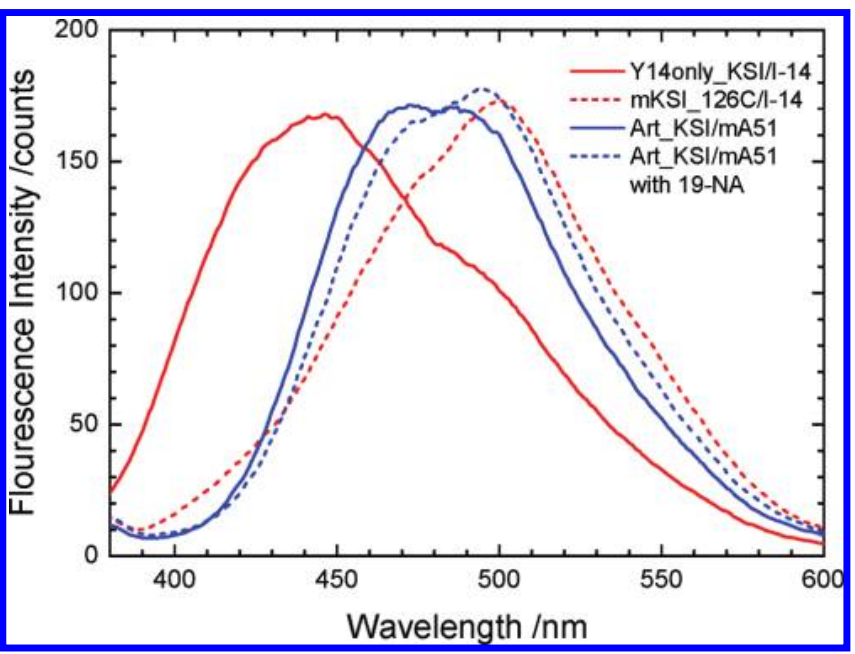

Figure 3. Fluorescence spectra of KSI mutants labeled with naphthalenesulfonic acid moiety. The fluorescence spectra of Y14only_KSI/ I-14 (solid red curve), mKSI_126C/I-14 (dotted red curve), Art_KSI/ mA51 (solid blue curve), and Art_KSI/mA51 treated 19-NA $(30 \mu \mathrm{M})$ (dotted blue curve) were exited at $335 \mathrm{~nm}$. All emission spectra were recorded for samples in Tris $-\mathrm{HCl}$ buffer $(50 \mathrm{mM}, \mathrm{pH} 7.5)$.

hydrophilic environment (Tris buffer), the emission was centered at $\sim 500 \mathrm{~nm}$, whereas the emission spectra shifted to $\sim 450 \mathrm{~nm}$ under hydrophobic conditions (IPA). This spectral shift thus serves as an indicator of the local environment in which the fluorophore is located.

In mKSI_126C/I-14, the only accessible site of reaction was Cys-126 of the protein; the emission spectrum is expected to be similar to that in Tris buffer. As Figure 3 shows, the emission spectrum of mKSI_126C/I-14 is centered at $\sim 500 \mathrm{~nm}$ with a shoulder at $\sim 475 \mathrm{~nm}$ likely due to an instrumental artifact (Woods anomaly). This result supports that the moiety of 5-[(2(acetamido)ethyl)amino]naphthalene-1-sulfonic acid was situated in a hydrophilic environment. The emission spectrum of Y14only_KSI/I-14 is centered at $\sim 450 \mathrm{~nm}$, similar to the spectrum of I-14 in IPA. This result indicates that the fluorophore was buried in a hydrophobic environment.

The steady-state fluorescence spectrum of Art_KSI/mA51 is centered at $\sim 475 \mathrm{~nm}$ (Figure 3). When we added 19-NA (30 $\mu \mathrm{M})$ to this system, the fluorescence emission shifted to become

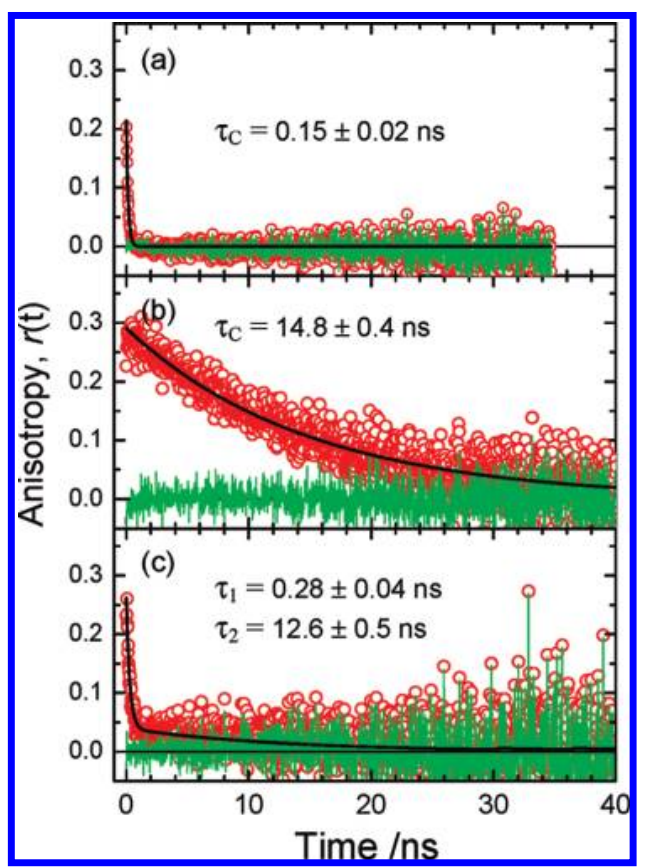

Figure 4. Fluorescence anisotropy transients of $\mathrm{I}-14$ obtained at $25^{\circ} \mathrm{C}$ : (a) I-14 in Tris buffer; (b) Y14only_KSI/I-14; (c) mKSI_126C/ I-14. Raw data are shown as open circles; fitted results are represented with solid curves.

centered at $\sim 500 \mathrm{~nm}$. The equilibrium between the ligand and the steroid-binding site was hence altered by competitive binding of 19-NA, and this change results in a greater distribution of mA51 being exposed to a polar hydrophilic environment, yielding the red-shifted emission.

3.4. Fluorescence Anisotropy Transients of I-14 in KSI Mutants. According to those steady-state results, the binding site of I-14 in Y14only_KSI was inside the hydrophobic cavity. To confirm this result, we measured time-resolved fluorescence anisotropy to obtain the depolarization kinetics for the rotational Brownian motion of the fluorophore in various biological environments. In a TCSPC experiment, the fluorescence anisotropy decay is evaluated with ${ }^{28,29}$

$$
r(t)=\frac{I_{\mathrm{VV}}(t)-G \times I_{\mathrm{VH}}(t)}{I_{\mathrm{VV}}(t)+2 \times G \times I_{\mathrm{VH}}(t)}
$$

in which the $G$ factor compensates for the polarization dependence of the monochromator grating, optics and detector. By definition, $G=I_{\mathrm{HV}} / I_{\mathrm{HH}}$, in which $I_{\mathrm{HV}}$ and $I_{\mathrm{HH}}$ represent the intensities of fluorescence excitation with horizontal polarized light and monitoring with vertical and horizontal polarizations, respectively; a typical result showing the $G$ factor of the system appears in Figure S2, Supporting Information. Analogously, IVV and $I_{\mathrm{VH}}$ represent the intensities of fluorescence excitation with vertically polarized light monitored with vertical and horizontal polarizations, respectively.

The fluorescence anisotropy decay curves of I-14 alone in Tris buffer, I-14 in Y14only_KSI, and I-14 in mKSI_126C at $25^{\circ} \mathrm{C}$ appear in Figure $4 \mathrm{a}-\mathrm{c}$, respectively. The observed timedependent curves in Figure 4 all decay to the background as zero at long times and are well described with a singleexponential (free isotropic rotation) or biexponential (free anisotropic rotation) decay function with separate rotational time coefficients. For I-14 in Tris buffer, the rotational time coefficient is $0.15 \mathrm{~ns}$, whereas for I-14 in Y14only_KSI, the rotational time coefficient increases to $14.8 \mathrm{~ns}$. The singleexponential decay feature of the system indicates a purely 
isotropic orientation relaxation that reflects the size of the system. According to Perrin's equation, the rotational correlation time coefficient $\left(\tau_{\mathrm{C}}\right)$ for a spherical molecule is proportional to the hydrodynamic molecular volume of the fluorophore $(V)$ and can be represented with ${ }^{29}$

$$
\tau_{\mathrm{C}}=\frac{V \eta}{R T}
$$

in which $\eta$ is the viscosity of the medium $(\eta=0.8937 \mathrm{mPa} \cdot \mathrm{s}$ at $298 \mathrm{~K}$ ), $T$ is the temperature (fixed at $298 \mathrm{~K}$ ), and $R$ is the gas constant. As a result, the hydrodynamic radii (assuming $V$ $=4 / 3 \pi r^{3}$ ) of I-14 alone in buffer and I-14 in Y14only_KSI are estimated to be 5.5 and $25.4 \AA$, respectively.

In the case of mKSI_126C/I-14, for which the fluorophore I-14 is expected to be labeled on the surface of the protein molecules, the fluorescence anisotropy transient had a biexponential decay with rotational time coefficients $0.28 \mathrm{~ns}$ (amp1 $=0.85)$ and $12.6 \mathrm{~ns}(\operatorname{amp} 2=0.15)$. The observed twocomponent feature is consistent with the rotational relaxation being due mainly to the local motion of the fluorophore outside the protein and rotation of the KSI, but the contribution of the former is much larger than that of the latter. Because I-14 was labeled on the surface of the protein, the rapid-decay component corresponding to the segmental motion of the fluorophore outside the protein is expected to become dominant in the observed depolarization curve. Furthermore, the rotational relaxation period of I-14 on the surface of mKSI_126C is substantially greater than that of free I-14 in Tris buffer solution ( 0.28 vs $0.15 \mathrm{~ns})$, indicating the significance of the restricted motion of the fluorophore that affects the observed depolarization kinetics.

3.5. Fluorescence Anisotropy Transients of Art_KSI/ mA51 in the Presence of 19-NA. The fluorescence anisotropy transient acts as a probe to estimate the rotational correlation time and the relative amplitude of the fluorophore in the protein system. Here we applied the anisotropy transients of mA51 in Art_KSI with 19-NA at varied concentrations to probe the interaction of 19-NA with Art_KSI and the relative fractions of mA51 inside and outside the steroid-binding site of Art_KSI. According to the results in Figure 4, the fluorophore in the steroid-binding site of the protein has an anisotropic behavior different from that labeled on the surface of KSI. As the fluorophore that is bound with a long chain (e.g., mA51) can alter its orientation between inside and outside the steroidbinding site in Art_KSI, the relative populations of fluorophoreKSI that exist in two environments display complicated kinetics. Figure 5 shows the anisotropy decays of mA51 in protein Art_KSI in the absence (Figure 5a) or presence (Figure $5 \mathrm{~b}-\mathrm{d}$ ) of additional analyte 19-NA. The anisotropy decays of Art_KSI/ mA51 exhibit a biexponential feature in the absence of 19-NA, but additional offset components are involved in the presence of 19-NA. As the magnitude of the offset signal increases consistently upon increasing the concentration of 19-NA, we infer that the rotational motion of the probe (mA51) was hindered by the presence of 19-NA in the binding site of Art_KSI. In the absence of 19-NA, the anisotropy decay shows only an anisotropic feature like the result shown in Figure 4c, but the system involves two contributions: one is the probe inside the protein pocket represented by $r_{\text {inside }}(t)$, and the other is the probe outside the protein pocket represented by $r_{\text {outside }}(t)$. As the former is described with a single-exponential decay function (isotropic rotation) and the latter described with a biexponential decay function with an offset (hindered anisotropic rotation), the observed time-dependent anisotropy, $r(t)^{\mathrm{obs}}$, is fitted satisfactorily with the following equation ${ }^{28}$

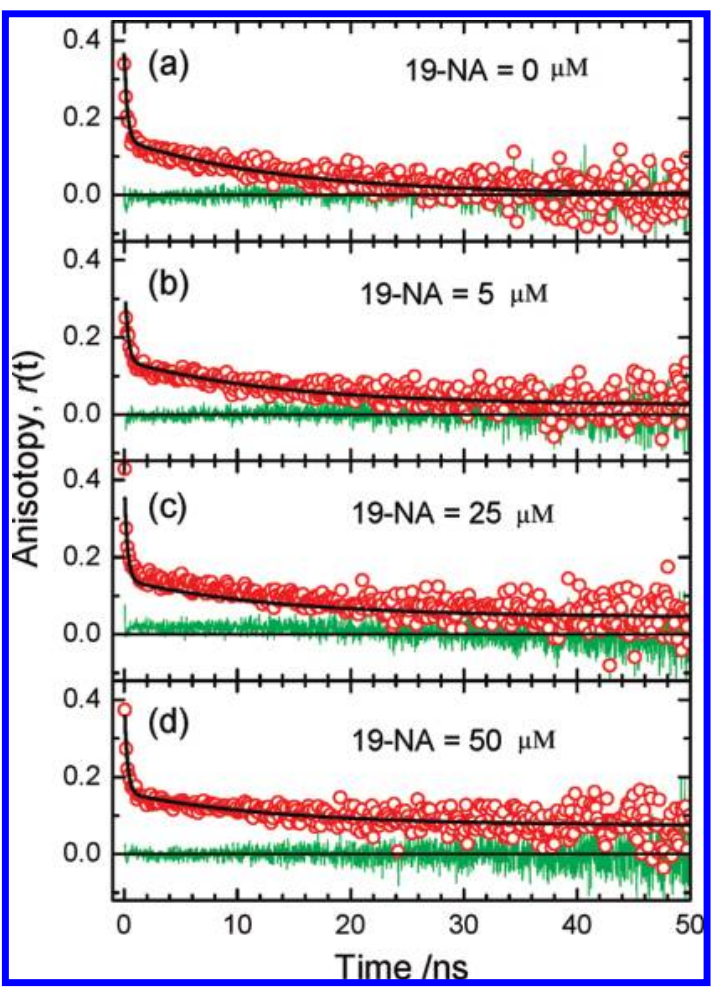

Figure 5. Fluorescence anisotropy transients of mA51 in Art_KSI excited at $375 \mathrm{~nm}$ and probed at $500 \mathrm{~nm}$ at $5{ }^{\circ} \mathrm{C}$ in presence of 19-NA $1 \mu \mathrm{M}$ (a) 0 , (b) 5, (c) 25, and (d) 50. Raw data are shown as red open circles; results fitted according to eq 3 are represented with black solid curves. The green traces correspond to the residuals of the fits.

$$
\begin{aligned}
& r(t)^{\text {obs }}=x \cdot r_{\text {inside }}(t)+(1-x) \cdot r_{\text {outside }}(t) \\
&=x \cdot r_{0}^{\text {in }} \times \exp \left(-t / \tau_{1}\right)+(1-x) \cdot \\
&\left\{( r _ { 0 } ^ { \text { out } } - r _ { \infty } ) \cdot \left[f \cdot \exp \left(-t / \tau_{2}\right)+\right.\right. \\
&\left.\left.(1-f) \cdot \exp \left(-t / \tau_{3}\right)\right]+r_{\infty}\right\}
\end{aligned}
$$

Parameters $\tau_{1}-\tau_{3}$ are the corresponding rotational correlation time coefficients, $r^{\mathrm{n}}$ and $r^{\mathrm{ut}}$ are the corresponding anisotropy values at time zero, $r_{\infty}$ is the anisotropy for a hindered rotation at infinite time, and $f$ is the weighting factor for the hindered rotation with rotational time $\tau_{2}$. Because the absorption coefficients at $\lambda=375 \mathrm{~nm}$ and the lifetimes monitored at $500 \mathrm{~nm}$ of orientations of the two types are the same, the observed timedependent anisotropy is a sum of these two components weighted by their corresponding contributions, i.e., $x$ and (1$x)$.

The physical meaning of eq 3 is that the anisotropy component results from the free rotation of a mA51 molecule inside Art_KSI characterized with a single-exponential anisotropy decay (decay coefficient $\tau_{1}$ ) to a background level, whereas the other component resulting from a hindered anisotropic rotation of a mA51 molecule outside Art_KSI is characterized with a biexponential anisotropy decay (decay coefficients $\tau_{2}$ and $\tau_{3}$ with relative amplitudes $f$ and $(1-f)$, respectively) to an asymptotic level, $r_{\infty}{ }^{28-30}$ Quantities $r_{0}^{\text {in }}$ and $r_{0}^{\text {out }}$ are fundamental anisotropy values of the two orientations, and $x$ and $(1-x)$ are the relative corresponding distributions of mA51 inside and outside the KSI cavity, respectively. According to the results obtained from the anisotropy transient of Y14only_KSI/I-14 at $25^{\circ} \mathrm{C}$ (Figure $4 \mathrm{~b}$ ), $r_{0}^{\text {in }}=0.286$, and $\tau_{1}$ is estimated to be 15.9 ns from eq 2 at $5{ }^{\circ} \mathrm{C}$. On addition of much 19-NA to saturate the steroid binding side of Art_KSI, most mA51, we assumed, is outside that binding site so that the condition $x=0$ is valid. 
TABLE 1: Fitted Parameters of Anisotropy Transients of Art_KSI/mA51 with Various Amounts of 19-NA Added to a Buffer Solution at $5^{\circ} \mathbf{C}^{a}$

\begin{tabular}{ccr}
\hline $19-\mathrm{NA} / \mu \mathrm{M}$ & $x$ & \multicolumn{1}{c}{$r_{\infty}$} \\
\hline 0 & $0.21 \pm 0.016$ & $0 \pm 0.001$ \\
5 & $0.098 \pm 0.045$ & $0.026 \pm 0.002$ \\
25 & $0.02 \pm 0.01$ & $0.046 \pm 0.002$ \\
50 & 0 & $0.074 \pm 0.003$
\end{tabular}

a The values of $x$ and $r_{\infty}$ were obtained from fitting the anisotropy data of Figure 5 according to eq 3 with the other parameters fixed as follows: $\tau_{1}=15.9 \mathrm{~ns}, \tau_{2}=0.27 \mathrm{~ns}, \tau_{3}=13.4 \mathrm{~ns}, f=0.74, r_{0}^{\text {in }}=$ 0.286 , and $r_{0}^{\text {out }}=0.40$.

Under such an assumption, the kinetic parameters for $r_{\text {outside }}$ are obtained on fitting the transients of Art_KSI/mA51 in the presence of 19-NA ( $50 \mu \mathrm{M})$ according to eq 3 , which yields $\tau_{2}$ $=0.27 \mathrm{~ns}, \tau_{3}=13.4 \mathrm{~ns}, f=0.74, r_{\infty}=0.074$, and $r_{0}^{\text {ut }}=0.40$. That $r_{0}^{\text {ut }}=0.40$ signifies that the Art_KSI/mA51 system with the probe completely outside the protein pocket features the absorption and emission dipoles in a parallel aligned geometry.

With parameters $r_{0}^{\text {in }}, r_{0}^{\text {ut }}, \tau_{1}-\tau_{3}$, and $f$ all fixed at the specified values, the anisotropy decays shown in Figure 5 are satisfactorily fitted according to eq 3 . The remaining fitting parameters $(x$ and $r_{\infty}$ ) are summarized in Table 1. Both parameters $x$ and $r_{\infty}$ exhibit a systematic variation as a function of the amount of added 19-NA. For Art_KSI/mA51 in the absence of 19-NA, $r_{\infty}$ $=0$; the system is hence characterized with a free anisotropic rotational motion. In the presence of 19-NA, however, the anisotropy transients decay to an asymptotic level $\left(r_{\infty}>0\right)$; this value increases systematically upon increasing the amount of 19-NA (Table 1). That $r_{\infty}$ remained nonzero reflects that the rotational motion of $\mathrm{mA5} 1$ outside the binding site was constrained by the presence of 19-NA. Moreover, as the observed $r_{\infty}$ is a statistical value that depends on the distribution of 19-NA bound in the binding site of Art_KSI, the value of $r_{\infty}$ increases when more $19-\mathrm{NA}$ is added to the Art_KSI/mA51 system. According to the wobble-in-cone model, ${ }^{28}$ the order parameter (i.e., the half an gle of the cone, $\theta_{\mathrm{C}}$ ) reflecting the degree of orientational constraint due to the presence of 19$\mathrm{NA}$ is determined with the following equation:

$$
\frac{r_{\infty}}{r_{0}^{\text {out }}}=\left[\frac{1}{2} \cos \theta_{\mathrm{C}}\left(1+\cos \theta_{\mathrm{C}}\right)\right]^{2}
$$

With $r r^{\text {ut }}=0.40$, the value of $\theta_{\mathrm{C}}$ is calculated to be $56^{\circ}$ under the saturated condition $\left(r_{\infty}=0.074\right)$ in which all probe molecules were outside the protein pocket and all binding sites were occupied by 19-NA.

The factor $x$ for the relative contribution is 0.21 in the absence of 19-NA, indicating that the fraction of mA51 inside the binding site of Art_KSI was $21 \%$ before addition of inhibitor. The distribution of mA51 outside the binding site of the protein is thus a major configuration, and the anisotropy results are consistent with the fluorescence spectra shown in Figure 3. The fluorescence spectrum of Art_KSI/mA51 (with a long paraffinic chain, 79\% outside) is similar to that of mKSI_126C/I-14 (with a short paraffinic chain, $100 \%$ outside) shown in Figure 3, but blue-shifted. We hence conclude that a long paraffinic chain makes mA51 more flexible so that the weak interaction between mA51 and the binding side of Art_KSI results in $79 \%$ of population with the probe outside the protein's binding site. The fluorescence spectrum of Art_KSI/mA51 with addition of 19NA shows a red-shifted spectral feature because the presence of 19-NA repels some mA51 from the hydrophobic binding site to a hydrophilic environment. The fraction of mA51 inside the protein decreased by half on addition of 19-NA $(5 \mu \mathrm{M})$, and to $2 \%$ with 19-NA $(25 \mu \mathrm{M})$.

\section{Conclusion}

A hydrophobic interaction prompted I-14 into a steroidbinding site in conjugation with Asp-38. This interaction between Y14only_KSI and I-14 and an identification of the site of I-14 labeling was confirmed with steady-state fluorescence and MS measurements. Art_KSI was constructed to evaluate the feasibility of a protein-ligand system for steroid detection. This steroid-recognition model was constructed by specifically conjugating the switching ligand to the Cys-86 of Art_KSI through formation of a disulfide bond. A comparison of the steady-state fluorescence of Y14only_KSI/I-14, mKSI_126C/ I-14, and Art_KSI/mA51 indicates the levels of the protein-ligand interaction in various environments. The steady-state fluorescence spectra demonstrate that 19-NA competed with mA51 for binding sites, and the distribution of mA51 in the steroidbinding site perturbed with 19-NA manifested with a red shift. With time-dependent fluorescence anisotropy of mA51 we probed the environment around it; the anisotropy revealed that the mA51 moiety on Art_KSI exists both inside and outside the steroid-binding cavity with $21 \%$ of mA51 inside the protein cavity. Addition of analyte 19-NA systematically decreased the fraction of mA51 inside the binding site of Art_KSI, providing direct evidence for the proposed steroid-recognition model in the engineered KSI system.

Acknowledgment. National Science Council of Taiwan and Ministry of Education of Taiwan, under the ATU program, provided support for this project.

Supporting Information Available: Mass spectrum of the I-14 labeling reaction of 14only_KSI (Figure S1a), LC separation of I-14-labeled peptide (Figure S1b), the $G$ factor of the TCSPC system (Figure S2), and sequences of primers to construct the Art_KSI gene (Table S1). This material is available free of charge via the Internet at http://pubs.acs.org.

\section{References and Notes}

(1) Chakrabarty, A.; Mallick, A.; Haldar, B.; Das, P.; Chattopadhyay, N. Biomacromolecules 2007, 8, 920.

(2) Mallick, A.; Haldar, B.; Chattopadhyay, N. J. Phvs. Chem. B 2005, $109,14683$.

(3) Houk, K. N.; Leach, A. G.; Kim, S. P.; Zhang, X. Angew. Chem.. Int. Ed. 2003, 42, 4872.

(4) Nakata, E.; Nagase, T.; Shinkai, S.; Hamachi, I. J. Am. Chem. Soc. 2004, 126, 490 .

(5) Takaoka, Y.; Tsutsumi, H.; Kasagi, N.; Nakata, E.; Hamachi, I. J. Am. Chem. Soc. 2006, 128, 3273.

(6) Tsukiji, S.; Wang, H.; Miyagawa, M.; Tamura, T.; Takaoka, Y.; Hamachi, I. J. Am. Chem. Soc. 2009, 131, 9046.

(7) Krishnamurthy, V. M.; Semetey, V.; Bracher, P. J.; Shen, N.; Whitesides, G. M. J. Am. Chem. Soc. 2007, 129, 1312.

(8) Wolthers, B. G.; Kraan, G. P. B. J. Chromatogr. A 1999, 843, 247.

(9) Ma, Y.-C.; Kim, H. Y. J. Am. Soc. Mass Spectrom. 1997, 8, 1010.

(10) Zarzycki, P. K.; Kulhanek, K. M.; Smith, R.; Clifton, V. L. J. Chromatogr. A 2006, 1104, 203.

(11) Kumar, A.; Aravamudhan, S.; Gordic, M.; Bhansali, S.; Mohapatra, S. S. Biosens. Bioelectron. 2007, 22, 2138.

(12) Li, G.; Liao, J. M.; Hu, G. Q.; Ma, N. Z.; Wu, P. J. Biosens. Bioelectron. 2005, 20, 2140.

(13) De, S.; Macara, I. G.; Lannigan, D. A. J. Steroid Biochem. Mol. Biol. 2005, 96, 235.

(14) Wu, Y.; Mitchell, J.; Cook, C.; Main, L. Steroids 2002, 67, 565. (15) Salvador, J.-P.; Sanchez-Baeza, F.; Marco, M.-P. Anal. Chem. 2007, 79, 3734

(16) Sheu, J.-T.; Chen, C. C.; Chang, K. S.; Li, Y.-K. Biosens. Bioelectron. 2008, 23, 1883. 
(17) Kuliopulos, A.; Mullen, G. P.; Xue, L.; Mildvan, A. S. Biochemistry 1991, 30, 3169.

(18) Xue, L.; Kuliopulos, A.; Mildvan, A. S.; Talalay, P. Biochemistry 1991, 30, 4991.

(19) Xue, L.; Talalay, P.; Mildvan, A. S. Biochemistry 1991, 30, 10858.

(20) Li, Y.-K.; Kuliopulos, A.; Mildvan, A. S.; Talalay, P. Biochemistry $1993,32,1816$.

(21) Wu, P.; Li, Y.-K.; Talalay, P.; Brand, L. Biochemistry 1994, 33 , 7415 .

(22) Kim, S. W.; Cha, S. S.; Cho, H.-S.; Kim, J.-S.; Ha, N.-C.; Cho, M.-J.; Joo, S.; Kim, K. K.; Choi, K. Y.; Oh, B.-H. Biochemistry 1997, 36, 14030 .

(23) Luo, L.-Y.; Chang, C.-H.; Chen, Y.-C.; Wu, T.-K.; Diau, E.W.-G. J. Phys. Chem. B 2007, 111, 7656.

(24) Yan, Y.; Marriott, G. Curr. Opin. Chem. Biol. 2003, 7, 635.
(25) Zechel, D. L.; Withers, S. G. Curr. Opin. Chem. Biol. 2001, 5, 643.

(26) Wan, C.-F.; Chen, W.-H.; Chen, C.-T.; Chang, M. D-T.; Lo, L.C.; Li, Y.-K. Biochem. J. 2007, 401, 551.

(27) Liu, S.-W.; Chen, C.-S.; Chang, S.-S.; Mong, K.-K.; Lin, C.-H.; Chang, C.-W.; Tang, C. Y.; Li, Y.-K. Biochemistry 2009, 48, 110.

(28) Valeur, B. Molecular Fluorescence: Principles and Applications; Wiley-VCH: New York, 2002.

(29) Lakowicz, J. R. Principles of Fluorescence Spectroscopv, 2nd ed.; Kluwer Academic/Plenum Publishers: New York, 1999.

(30) Chang, C.-W.; Lu, Y.-C.; Wang, T.-T.; Diau, E. W.-G. J. Am. Chem. Soc. 2004, 126, 10109.

JP908509W 